\title{
CONTROL PRENATAL Y SU IMPACTO EN LA MORTALIDAD MATERNA. ANÁLISIS DE UNA TENDENCIA, 1994-2004. CALI, COLOMBIA
}

\section{Prenatal controll and its impact on reducing maternal deaths. Trend anallysis, 1994-2004. Cali, Colombia}

\author{
Sonia Pazmiño de Osorio, M.D., * Ney Guzmán-Gómez, M.D., Ph.D.** \\ Recibido: diciembre 5/07 - Aceptado: febrero 2/09
}

\section{RESUMEN}

Objetivo: analizar el comportamiento de las razones de mortalidad materna en la ciudad de Cali (Colombia) durante dos décadas, 1985 a 2004, a la luz de indicadores de desarrollo social y organización de los servicios de atención a la gestante.

Metodología: se utiliza como marco conceptual el modelo canadiense propuesto por Laframboise y Lalonde en 1974, para explicar la contribución de los servicios de salud en la disminución de las tasas de mortalidad materna.

Resultados: a partir de 1994 se inició un descenso sostenido de la mortalidad materna, que se ha mantenido hasta el 2004. Durante este período no se evidenció un mejoramiento cuantitativo significativo de los indicadores de desarrollo social y económico de la ciudad de Cali. Las altas coberturas de atención prenatal (97\%) y del parto institucional (98\%), junto con un sistema eficiente de referencia y contrareferencia, se mantuvieron estables.

El control de los procesos de mejoramiento de la calidad de la atención prenatal, con evaluaciones y ajustes periódicos, y la participación permanente de ginecobstetras en el nivel primario de atención,

* Ginecobstetra, Especialista en Salud Pública, Administrador de Calidad. Universidad del Valle. Cali, Colombia. Correo electrónico: soniapacosta@hotmail.com

** M.D., Ph.D. Escuela de Salud Pública. Universidad del Valle. Cali, Colombia. constituyeron los hitos primordiales de las intervenciones realizadas en la red de servicios públicos de atención.

Discusión: los factores de riesgo intervenidos en la atención prenatal, la alta cobertura institucional de atención del parto y una red de referencia y contrareferencia de pacientes, oportuna y eficiente, parecen ser responsables de la disminución del 80\% de las muertes, según el cálculo de Guzmán en 1986.

Palabras clave: maternidad segura, garantía de calidad, calidad de atención a la gestante, control prenatal, participación de los servicios de salud, mortalidad materna, investigación evaluativa.

\section{SUMMARY}

Objective: analysing the statistical data regarding maternal deaths in Cali (Colombia) during two consecutive decades (1985-2004) and correlating outcomes with environmental and social indicators. Methodology: the Canadian Laframboise conceptual model was used for explaining how health service organisation and other aspects of medical care play a part in decreasing maternal mortality rates.

Results: maternal deaths in Cali have been decreasing since 1994. No significant quantitative variations in environmental and/or social indicators for Cali were detected from 1994-2004. High prenatal control (97\%) and institutional delivery coverage (98\%) 
remained stable, together with an efficient referral and counter-referral system. Improving prenatal attention quality through periodic evaluation and adjustment and obstetrician/gynaecologists' ongoing participation in first-level attention were the highlights of public attention service network intervention.

Discussion: prenatal risk factors, broad institutional coverage for a quality motherhood care programme and an efficient referral system have all contributed towards reducing maternal deaths by nearly 80 (taking Guzmán’s 1986 calculation as reference point).

Key words: safe motherhood, safe maternity, quality assessment, prenatal care, maternal death, evaluative research.

\section{INTRODUCCIÓN}

Hasta 1994, las razones de mortalidad materna en la ciudad de Cali, Colombia (2.423.381 habitantes) se mantuvieron estables y elevadas: 88,7 x 100.000 nacidos vivos; ${ }^{1}$ pero a partir de este año, se observó una tendencia decreciente que alcanzó el valor de 19,0 x 100.000 (figura 1). Este resultado contrasta con la situación de muertes maternas en el país: 104,9 x 100.000, ${ }^{2}$ cifra 17 veces mayor que la encontrada en países desarrollados y cuatro veces mayor que la de países con producto interno bruto similar. ${ }^{3}$

La contabilidad de las muertes en Cali es confiable, pues la Secretaría de Salud dispone de un sistema de vigilancia epidemiológica de muertes maternas, que data desde 1986, el cual permite rastrear con bastante precisión, no sólo el número de muertes por año, sino la causa básica de la defunción y otra información sociodemográfica. En la década de 1994 a 2004 las muertes maternas, en cifras absolutas, pasaron de 35 a 8, siendo las principales causas de muerte la preeclampsia, las hemorragias, el parto y postparto, los abortos y otras complicaciones del embarazo. La mayoría de estas muertes son consideradas evitables, ${ }^{4}$ a juicio del Comité de Mortalidad Materna Municipal.
Figura 1. Tendencia de la mortalidad materna en Cali (razones por cien mil nacidos vivos), 1985-2004.

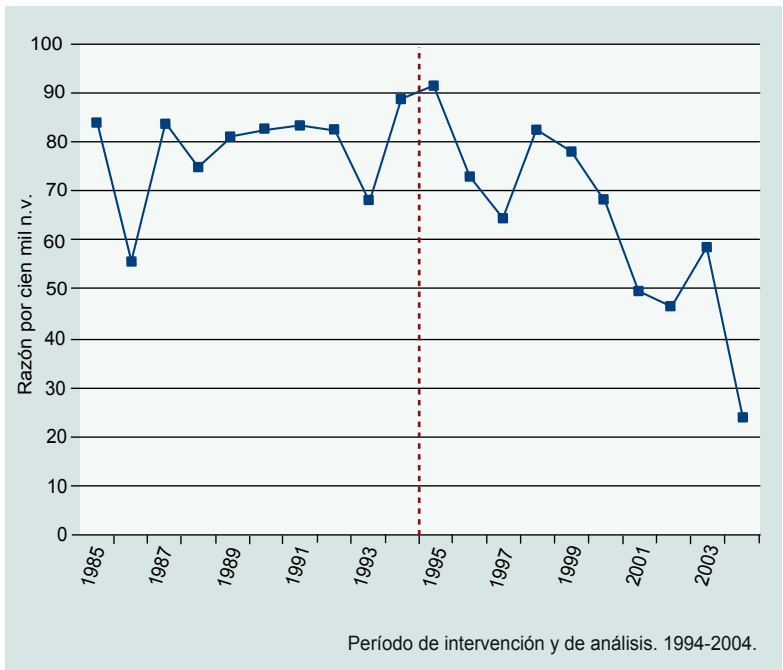

Dado que existe poca evidencia en la literatura médica acerca de la influencia del control prenatal en la reducción de la mortalidad materna, ${ }^{5}$ se realizó esta investigación evaluativa de procesos y resultados, con el objetivo de analizar el comportamiento de la mortalidad materna en la ciudad de Cali, Colombia durante dos décadas, 1985 a 2004, a la luz de indicadores de desarrollo social y de organización de los servicios de atención a la gestante, que de manera simultánea con un programa de intervención a la calidad de la atención prenatal, pudieran haber influido en su descenso.

\section{METODOLOGÍA}

Para el análisis realizado se utilizó el concepto de "campo de la salud", propuesto inicialmente por Laframboise $^{6}$ en 1973, y desarrollado más tarde por Lalonde y Alan Dever.,

Este concepto indica que la salud está determinada por una variedad de factores que se pueden agrupar en cuatro categorías primarias: estilo de vida, medio ambiente, organización de la atención a la salud y biología humana. Este arreglo conceptual permite ubicar los factores de riesgo y las estrategias específicas necesarias para evitar la enfermedad, siendo la preservación de la salud (promoción de la 
salud, prevención social y prevención de la enfermedad) su fundamento. Este modelo fue adoptado por el gobierno de los Estados Unidos, en su "perfil preventivo", anexo al programa de salud en 1980. El riesgo atribuible global por categorías ha sido establecido por un consenso de expertos para la mayoría de las enfermedades conocidas. ${ }^{9}$ Es especialmente útil cuando el período de observación es corto y la población de una alta vulnerabilidad, como es el caso de las mujeres embarazadas.

Guzmán ${ }^{10}$ calculó las fracciones etiológicas atribuibles para la mortalidad por todas las causas, según las 4 categorías primarias, para Cali en 1986. Encontró que los servicios de salud que se presten a las embarazadas y se cumplan con una eficacia del 100\%, podrán disminuir la mortalidad en un 60\%; otro 20\% adicional se podrá lograr con programas de promoción de la salud y prevención de la enfermedad, que mejorarán estilos de vida y ambiente. Por lo tanto, y dada la relevancia de los hallazgos anteriores, se decidió enfocar el análisis en dos categorías: medio ambiente y organización de la atención a la salud durante la última década (1994-2004), período durante el cual se presentó la reducción en la mortalidad materna.

\section{Procedimiento}

Se realizó una revisión documental de estadísticas socioeconómicas y sanitarias, reformas administrativas de los servicios, encuestas de morbilidad y mortalidad materno infantil, proyectos de promoción y prevención en salud sexual y reproductiva, ejecutados en el período, y algunas disposiciones legales que pudieron afectar la gestión municipal, en especial la prestación de los servicios de salud. Igualmente, se revisaron los registros y actas del Comité de Mortalidad Materna Municipal, y se entrevistaron funcionarios del nivel directivo de las Secretarías de Salud Municipal y Departamental, a través de entrevistas no estructuradas, requiriendo su percepción personal sobre lo ocurrido en la última década.
En el análisis estadístico se utilizó un modelo de regresión múltiple y se calculó el coeficiente de determinación, para estimar la contribución de las comunas con mayor mortalidad materna, al descenso de la mortalidad municipal, después de ser intervenidas con los procesos de mejoramiento de la calidad de atención prenatal.

\section{RESULTADOS}

En la categoría de medio ambiente se analizó el contexto legislativo y algunos factores ambientales, demográficos y de desarrollo económico del municipio.

\section{Contexto legislativo}

En este contexto se puede mencionar la expedición de la ley 100 de 1993, por la cual se creó el Sistema Nacional de Seguridad Social en Salud. Esta ley enmarcó la organización y funcionamiento de los servicios de salud.

A nivel local, el acuerdo 106 de 2003, del Concejo Municipal de Cali, descentralizó la prestación de los servicios de salud al primer nivel de atención, mediante la creación de cinco Empresas Sociales del Estado (ESE) y asignó a cada una de ellas la población correspondiente de áreas geográficas circunvecinas; determinó sus ingresos, su estructura básica y sus órganos de dirección y servicios.

\section{Factores ambientales, demográficos y de desarrollo económico del municipio, que influyeron en la salud materna}

La ciudad de Cali se vio afectada en el período de análisis, por alta migración de población rural desplazada por la violencia de otras ciudades y departamentos. ${ }^{11}$

Las mujeres en edad fértil, la población obstétrica y los nacidos vivos vienen aumentando progresivamente. La tasa de natalidad por mil habitantes era de 21,23 para el 2003. La tasa global de fecundidad continuaba disminuyendo $(2,07)$, mientras que el porcentaje de analfabetismo ha permanecido estable con $4,8 .^{12}$ 
La tasa de desempleo, que en 1994 fue 10,3\%, se incrementó a 15,6\% en el 2004, con un subempleo de $36,1 \%$, ambas cifras por encima del promedio nacional. El porcentaje de personas en situación de pobreza (1994 y 1998) se incrementó en 9 puntos porcentuales y la proporción de población en situación de indigencia aumentó en 5 puntos. En el período de 1998 a 2004, el porcentaje de pobreza pasó de $39,0 \%$ a $67,5 \%$ de la población, en tanto que la indigencia se incrementó de $10,0 \%$ a $34,8 \%$ en 2004. ${ }^{13}$

Podría decirse que el entorno socio-económico de la ciudad se deterioró durante la década de 1994 a 2004 y que las condiciones nutricionales y de salud de las gestantes no se favorecieron.

\section{Factores de los servicios de salud. Cobertura y atención}

La cobertura en atención prenatal (97,1\% - 97,2\%) y del parto institucional $(94,9 \%-98,8 \%)$ permanecieron altas durante la década. ${ }^{14}$

La atención prenatal y del parto en la ciudad, para la población pobre, se realizó en centros y hospitales de nivel primario, atendidos por un médico general y una enfermera a partir de 1998, y las complicaciones se remitieron a través de un sistema de referencia mediado por un Centro Regulador de Urgencias, a hospitales de mayor complejidad que funcionan con médicos especialistas y que son fácilmente accesibles.

En cuanto a los proyectos y programas de salud sexual y reproductiva, realizados por la Secretaría de Salud, ninguno tuvo evaluación de resultados en la salud materna y perinatal.

De esta manera, el problema de mortalidad materna parece concentrarse prioritariamente en la calidad de la atención. Según el comité de mortalidad materna, alrededor del 80\% de las muertes fueron calificadas como "evitables" y se demostró que la atención prenatal no fue la mejor al comenzar la intervención de la Secretaría de Salud Municipal, en alianza con la Asociación Vallecaucana de Obstetricia y
Ginecología con los procesos de mejoramiento de la atención a la gestante en la década de 1994 a $2004 .^{15,16}$ La calidad mejoró durante la intervención.

Algunas ineficiencias detectadas fueron: la falta de detección del riesgo por enorme variabilidad en las técnicas de medición, sólo una de cada dos pacientes fue examinada completamente y no se realizaron exámenes ginecológicos en la mayoría de ellas; el incumplimiento de las normas se estimó en un 35\%; y hubo insuficiencia en la capacidad resolutiva de los servicios. Existía, además, gran desmotivación en el personal y falta de interés y compromiso de los directivos y funcionarios.

La situación encontrada fue objeto de análisis y reflexión de manera participativa con todo el personal asistencial y administrativo; se motivó sobre la importancia de mejoramiento continuo para procurar la salud materna y del recién nacido. Una actualización de los conocimientos y las prácticas, junto con un sistema de control estricto, con participación de ginecobstetras, indicadores de estructura, proceso y resultados, complementaron el proceso.

El seguimiento de más de 10.000 gestantes demostró una reducción de la morbilidad materna (ruptura prematura de membranas y desproporción cefalopélvica disminuyeron en un 64,9 y $86,0 \%$, respectivamente) y perinatal (las patologías del recién nacido decrecieron en un $86,0 \%)$. No ocurrieron muertes maternas atribuibles al control prenatal y la mortalidad fetal bajó en un $76,0 \%{ }^{17}$

En la década de 1994 a 2004 se presentó un descenso paulatino de la mortalidad materna en el municipio, correlacionado con el observado en las zonas de influencia de las ESE intervenidas con los procesos de mejoramiento de la calidad en la atención. En dos de ellas (comunas 7 y 14) se estimó su contribución de 30\% al descenso global, según el coeficiente de determinación (tabla 1 y figura 2). 


\begin{tabular}{|c|c|c|c|c|c|c|c|}
\hline AÑO & $\begin{array}{c}\text { Nacidos } \\
\text { vivos Cali }\end{array}$ & $\begin{array}{l}\text { Muertes } \\
\text { maternas }\end{array}$ & $\begin{array}{l}\text { Razón de } \\
\text { mortalidad }\end{array}$ & $\begin{array}{c}\text { Nacidos } \\
\text { vivos } \\
\text { comuna } 14\end{array}$ & $\begin{array}{c}\text { Muertes } \\
\text { esperadas }\end{array}$ & $\begin{array}{c}\text { Muertes } \\
\text { observadas }\end{array}$ & $\begin{array}{c}\text { REM } \\
\text { Razón } \\
\text { estandarizada } \\
\text { mortalidad } \\
\text { materna }\end{array}$ \\
\hline 1994 & 40.575 & 36 & 88,7 & 2816 & 2,5 & 5 & 2 \\
\hline 1995 & 41.917 & 36 & 85,8 & 2915 & 2,5 & 5 & 2,0 \\
\hline 1996 & 42.581 & 29 & 68,1 & 2968 & 2,0 & 5 & 2,5 \\
\hline 1997 & 43.127 & 25 & 57,9 & 3013 & 1,7 & 5 & 2,9 \\
\hline 1998 & 41.195 & 34 & 82,5 & 3168 & 2,6 & 4 & 1,5 \\
\hline 1999 & 41.864 & 33 & 78,8 & 3224 & 2,5 & 3 & 1,2 \\
\hline 2000 & 42.541 & 28 & 65,8 & 3281 & 2,2 & 3 & 1,4 \\
\hline 2001 & 43.225 & 20 & 46,3 & 3338 & 1,5 & 2 & 1,3 \\
\hline Total & 337.025 & 241 & 71,5 & 24.723 & 17,7 & 32 & 1,8 \\
\hline
\end{tabular}

Períodos de intervención

IC 95\%; 26,7 - 92,6

Modelo de regresión múltiple. Coeficiente de determinación comuna 14: 27\%

Coeficiente de determinación para las comunas 7 y 14: 30\%.

Figura 2. Tendencia del número de muertes maternas por ESE. 1994 - 2004. Cali

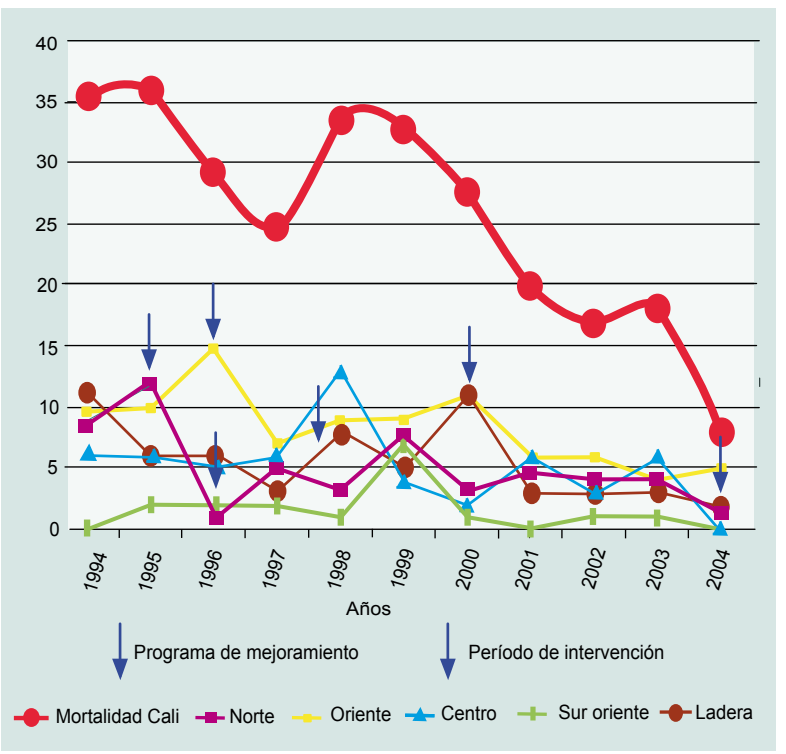

\section{DISCUSIÓN}

Identificar específicamente los factores responsables del descenso de la mortalidad, en ausencia de estudios evaluativos específicos, es una tarea bastante compleja.
La razón de mortalidad materna es un indicador que refleja el grado de desarrollo de los países; sin embargo, algunos países en desarrollo presentan tasas de mortalidad bajas, lo cual han logrado con un compromiso político sólido y modelos de atención médica específicos, como son los casos de Cuba y Sri Lanka. ${ }^{18}$

Aunque existe poca evidencia en la literatura médica acerca del poder del control prenatal en la reducción de la mortalidad materna, ${ }^{5} \mathrm{y}$ teniendo en cuenta la poca variación de las altas coberturas de atención prenatal y parto institucional, así como el mantenimiento de un statu quo de las condiciones de desarrollo social y económico, con excepción del progreso en la malla vial de la ciudad, que pudo haber mejorado la accesibilidad de los servicios de salud, el descenso de las razones de mortalidad materna, a diferencia de otras poblaciones con desarrollos socioeconómicos similares (figura 3), puede atribuirse a la eficacia y efectividad del programa de mejoramiento de la calidad de la atención prenatal. 
Figura 3. Razón de mortalidad materna en Cali, Medellín, Valle, Antioquia y Risaralda. 1994 - 2004

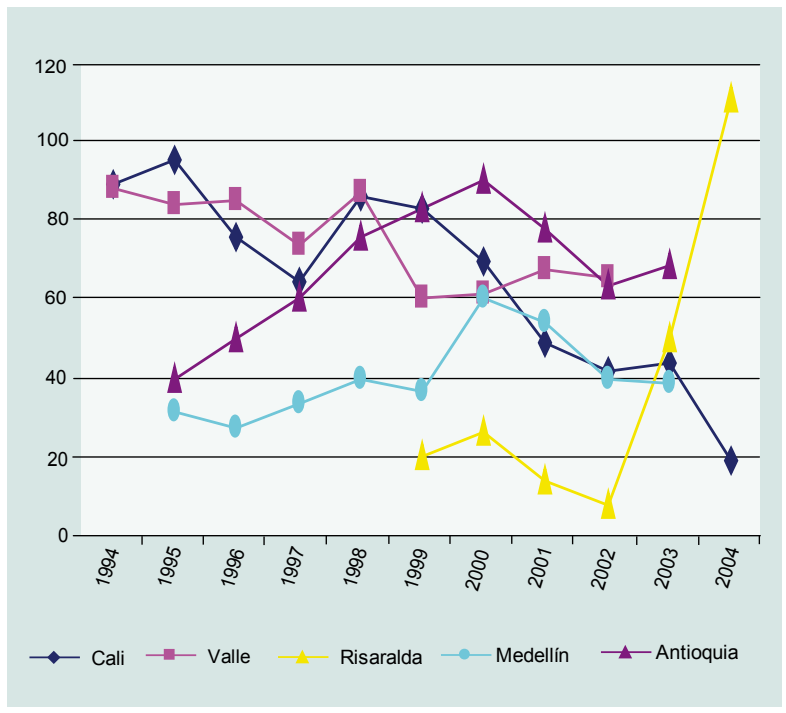

De esta forma, las estimaciones propuestas por Laframboise $^{6}$ y Guzmán ${ }^{10}$ sobre la contribución de la organización de los servicios a la mortalidad materna y el programa de mejoramiento de la calidad de la atención a la gestante, respaldan la importancia de generar conciencia para mejorar la calidad de los servicios y realizar evaluaciones periódicas con indicadores de resultado en la salud materna y perinatal.

La evaluación será más productiva cuando constituya un proceso continuo que alimente constantemente a los administradores, coordinadores del programa y gerentes a cargo de la toma de decisiones. Así mismo, la evaluación debe alertar a los funcionarios sanitarios acerca de las tendencias en la prestación de los servicios y señalar los problemas que requieren una acción correctiva.

El sistema de control periódico establecido en los procesos de mejoramiento de la calidad de la atención prenatal, con la participación de ginecobstetras en el nivel primario, se considera un avance dentro del sistema actual de atención nacional.

Finalmente, en respaldo a las conclusiones presentadas, vale la pena citar a Jahn \& DeBrouwere ${ }^{18}$ sobre las precondiciones para la disminución de la mortalidad materna en los países industrializados: "Tener conciencia de la magnitud del problema, reconocer que la mayoría de las muertes maternas son evitables y promover la movilización de los profesionales y de la comunidad. Si países con comparable riqueza o pobreza tienen diferentes mortalidades maternas, esto puede deberse no sólo a la disponibilidad de servicios, sino también a la calidad de los mismos, y a la manera como se relacionan con los pacientes. Cuando uno puede combinar acceso a un cuidado primario "de calidad" y referir las pacientes para la atención del parto, las razones de mortalidad materna pueden caer relativamente en corto tiempo". Esta aseveración ha sido demostrada en diferentes estudios. ${ }^{19}$

\section{REFERENCIAS}

1. Secretaría de Salud Pública Municipal. Mortalidad materna. Cali, 1985-1993. Boletín epidemiológico. Santiago de Cali; 1993.

2. Ministerio de la Protección Social. República de Colombia. Plan de choque para la reducción de la mortalidad materna. Bogotá, D.C. Ago; 2004.

3. Population Reference Bureau. Las mujeres de nuestro mundo. Washington; 2005.

4. Secretaría de Salud Municipal. Archivo Comité Mortalidad Materna Municipal. 1984-1994.

5. World Health Organization. Antenatal care in developing countries. Promises, achievements and missed opportunities. An analysis of trends, levels and differentials, 1990-2001. WHO; 2003.

6. Laframboise HL. Health policy: Breaking the problem down in more manageable segments. Canadian Medical Association Journal 1973;108:388-91.

7. Lalonde M. A new perspective on the health of canadians. Ottawa: Information Canada Health and Welfare; 1974.

8. Dever GEA. Community health analysis. Rockville, M.D.: Aspen System Corporation; 1980.

9. Dever GEA. An epidemiological model for health policy analysis. Soc Indicators Res 1976;2:452-66.

10. Guzmán N. Hacia una nueva política de salud en Colombia. 2da. Edición. Cali: Feriva Editores; 1992.

11. Diagnóstico situacional de la población en situación de desplazamiento-PSD-asentada en el municipio 
de Cali. Oficina Gestión de Paz y Convivencia de la Gobernación del Valle, en convenio con OIM; 2005.

12. Duque S. División de Estadística Secretaría de Salud Pública Municipal de Cali.

13. Secretaría de Salud Pública Municipal- CEDETES. Construcción de un modelo de salud para Cali. Línea de base para la formulación del modelo. CEDETES BOLETÍN_01 Santiago de Cali; 2007.

14. Profamilia. Salud Sexual y Reproductiva en Colombia. Encuesta Nacional de Demografía y Salud. 2000-2005. Bogotá, Colombia.

15. Pazmiño S. Mejoramiento continuo de la calidad de atención. Rev Colomb Obstet Ginecol 1997;48:39-46.
16. Pazmiño S, Amashta F, Perdomo C, Torres LE, Rojas J, Arturo MC, et al. La calidad del control prenatal como garantía de salud de madres y recién nacidos. Rev Colomb Obstet Ginecol 1998;49:190-5.

17. Pazmiño S, Guzmán N. Mejoramiento continuo de la atención a la gestante. Asociación Vallecaucana de Obstetricia y Ginecología. Cali: Artes Gráficas del Valle Ltda; 2002.

18. Brouwere V, Lerberghe W. Safe motherhood Strategies: a review of the evidence; 2001.

19. Kassas M, Campbell O. The National Maternal Mortality Study of Egypt 1992-1993. Int J Gynaecol Obstet 1995;50:101-8. 